Reutner - Der effiziente Staat - Fiktion oder Vision? 
Friedrich Reutner

\title{
Der effiziente Staat - Fiktion oder Vision?
}

\author{
Unternehmerische Konzepte \\ für Lebensqualität und Wohlstand
}

3., ergänzte Auflage 
Die Deutsche Bibliothek - CIP-Einheitsaufnahme

\section{Reutner, Friedrich:}

Der effiziente Staat: Fiktion oder Vision? : unternehmérische

Konzepte für Lebensqualität und Wohlstand / Friedrich Reutner.

-3., erg. Aufl. - Wiesbaden : Gabler, 1997

ISBN 978-3-322-82936-8

Alle Rechte vorbehalten

(C) Betriebswirtschaftlicher Verlag Dr. Th. Gabler GmbH, Wiesbaden 1997

Lektorat: Ulrike M. Vetter

Softcover reprint of the hardcover 3rd edition 1997

Der Gabler Verlag ist ein Unternehmen der Bertelsmann Fachinformation GmbH.

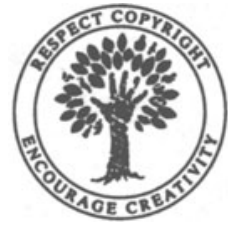

Das Werk einschließlich aller seiner Teile ist urheberrechtlich geschützt. Jede Verwertung außerhalb der engen Grenzen des Urheberrechtsgesetzes ist ohne Zustimmung des Verlags unzulässig und strafbar. Das gilt insbesondere für Vervielfältigungen, Übersetzungen, Mikroverfilmungen und die Einspeicherung und Verarbeitung in elektronischen Systemen.

http://www.gabler-online.de

Höchste inhaltliche und technische Qualität unserer Produkte ist unser Ziel. Bei der Produktion und Verbreitung unserer Bücher wollen wir die Umwelt schonen: Dieses Buch ist auf säurefreiem und chlorfrei gebleichtem Papier gedruckt. Die Einschweißfolie besteht aus Polyäthylen und damit aus organischen Grundstoffen, die weder bei der Herstellung noch bei der Verbrennung Schadstoffe freisetzen.

Die Wiedergabe von Gebrauchsnamen, Handelsnamen, Warenbezeichnungen usw. in diesem Werk berechtigt auch ohne besondere Kennzeichnung nicht zu der Annahme, daß solche Namen im Sinne der Warenzeichen- und Markenschutz-Gesetzgebung als frei zu betrachten wären und daher von jedermann benutzt werden dürften.

Umschlaggestaltung: Schrimpf und Partner, Wiesbaden

Satz: FROMM MediaDesign GmbH, Selters/Ts. 


\section{Inhalt}

1. Lebensqualität als Ziel der Wirtschaftspolitik

Die Bedeutung der Wirtschaft und die Bedürfnisstruktur im Wandel

Das richtige $\mathrm{Maß}$ an motivierendem Druck und Erfolgserlebnis

Leistungskulturen steigern Zufriedenheit und Erfolg administrierte Kulturen senken die Lebensqualität und steigern die Korruption Streitkulturen senken die Lebensqualität und den Lebensstandard

Korruption, Kriminalität und Unpünktlichkeit sind wichtige Faktoren der Unwirtschaftlichkeit und Lebensqualität

Das Verbesserungspotential ist groß

2. Die Qualität der Führung entscheidet langfristig über Erfolg und Mißerfolg

Die Führung prägt die Kultur, die Kultur prägt die Bürger _ 39

Eine Selbstmordkultur zerstört die wirtschaftliche Basis __ 43

Die Verlockung, ins Detail einzugreifen _ 48

Beurteilung der Managementqualität _ 51

Sinkt die Qualität der Manager? _ 52

Administration verdirbt das Management $\quad 54$

Die Rückdelegation lenkt vom Wesentlichen ab 55

Aus Unternehmern werden Verwalter _ 57

Sind Beamte schlechter als Manager? _ 60

Voraussetzungen in der Politik __ 62

Managementmethoden und -untersuchungen weisen den Weg zur Leistungsstärke 
Managementmethoden machen Organisationen

leistungsfähiger 66

Erfolgsfaktoren verbessern Erlöse und Gewinnsituation _ 68

3. Strukturveränderung: Gefahr und Chance

Strukturprobleme führen zum Ungleichgewicht

Die langfristige Betrachtung entscheidet

Die drei Strukturzustände: positives Ungleichgewicht,

Gleichgewicht und negatives Ungleichgewicht

Unternehmensanalysen verdeutlichen

die wirtschaftlichen Zusammenhänge

Die lange Inkubationszeit von Strukturkrisen

Der Trend zum negativen Ungleichgewicht

Die wirtschaftlich optimierte Zwangskonstellation 86

Eine falsch orientierte Zwangskonstellation und monopolistische „Ventile“ führen zur Unwirtschaftlichkeit

Einige Beispiele für hochgradige Wertvernichtung

Der Teufelskreis falscher Gesetze

Die Signale nachlassender struktureller Wettbewerbsfähigkeit 108

Die Verwaltung ist zu groß

Die Steuerungsinstrumente zur Wirtschaftlichkeit

funktionieren nicht

Überkomplexität als Strukturproblem

Kreativität und Leistungskultur verfallen

Die Innovationskraft sinkt

Der tertiäre Sektor als Alarmsignal

\section{Leistungskultur: Basis der Wettbewerbsfähigkeit}

Wirtschaftlicher Erfolg basiert zu mehr als der Hälfte auf Psychologie

Unternehmenskultur und volkswirtschaftliche Kultur

Leistungskulturen erfordern viele Voraussetzungen

Klare Ziele sind die Voraussetzung für die Entwicklung einer Leistungskultur

Interessenharmonie als Grundlage der Leistungskultur _ 150

Überschaubare Regeln, Freiheit im Detail, Strenge

in den Grundwerten 
Wertvernichtende Administration und Zentralisierung gefährden Leistungskulturen

Ohne Leistungskulturen gibt es kaum Kreativität _ 169

Negative Kulturen sind schwer zu verändern

5. Kultur- und Strukturprobleme erzeugen Arbeitslosigkeit, Investitionsdefizite und Verschuldung

Der falsche Ansatz zur Beseitigung der Arbeitslosigkeit __ 176

Strukturprobleme und Arbeitslosigkeit _ 180

Strukturprobleme und Investition 188

Strukturprobleme und Verschuldung 190

Die Relation im Wettbewerb entscheidet _ 195

\section{Ansatzpunkte für geänderte Rahmenbedingungen}

Durch Benchmarking erkennt man optimierte Strukturen und Kulturen

Determinanten des wirtschaftlichen Erfolges

einer Volkswirtschaft

Umfassende motivierende Zwangskonstellation erzeugen 207

Qualität der wirtschaftspolitischen Führung erhöhen 211

Die Erfolgsformel kennzeichnet den Gleichgewichtszustand, der Gleichgewichtszustand führt zur bestmöglichen Wohlstandsentwicklung

Kurzfristiges Gleichgewicht herstellen

Mittel- und langfristiges Gleichgewicht steigert

den Lebensstandard

Wie erreicht man die günstigste Beschäftigungssituation?

Die Erfolgsformel erklärt den Auf- und Abstieg

Entfaltung der Leistungskultur führt

zur wirtschaftlichen Dynamik

Erste Bedingung: Leistungssteigernde wirtschaftliche

Zwänge führen zur eigenen Wunscherfüllung

Zweite Bedingung: Allgemein bekannte Ziele

und Impulse stützen die Leistungskultur

Dritte Bedingung: Zum Aufbau einer Leistungskultur gehört vor allem, daß Erfolge gemessen

und honoriert werden 